\title{
Aufsätze
}

\section{Das Buch vom Tempel und verwandte Texte Ein Vorbericht}

\section{Joachim Friedrich Quack/Berlin}

Ziel des nachfolgenden Beitrages ist es, einen relativ ausführlichen Vorbericht über einen umfangreichen ägyptischen Text zu geben, der detaillierte Angaben zum Tempelbetrieb im Alten Ägypten bietet. Dieser Text, den ich als das „Buch vom Tempel " bezeichnen möchte, ist als ganzer noch unveröffentlicht und soll von mir in absehbarer Zeit in monographischer Bearbeitung vorgelegt werden. ${ }^{1}$ Hier sollen seine wesentlichen Züge vorgestellt werden, wobei der Schwerpunkt auf der reinen Materialpräsentation liegt. Die zusammenhängende Auswertung und Kommentierung muß der Endpublikation vorbehalten bleiben.

Das Buch vom Tempel ist ein ausgesprochen reich bezeugter Text. Derzeit sind mir etwa vierzig verschiedene Handschriften bekannt, die in etliche hundert Einzelfragmente zerfallen sind. Von diesen sind die meisten noch unpubliziert oder allenfalls mit knappen und oft ungenauen Angaben beschrieben worden. Herkunftsorte sind, soweit bekannt, Tebtynis und Dime im Fayum sowie Elephantine. Man kann demnach annehmen, daß der Text im ganzen Land bekannt war, und die Menge der an einem Ort gefundenen Handschriften ${ }^{2}$ deutet auf seine praktische Bedeutung hin. Ungeachtet der scheinbar sehr reichen Bezeugung ist der Text alles andere als vollständig rekonstruiert, da die meisten Handschriften in sehr schlechtem Erhaltungszustand sind. Bïsher dürfte nur etwas über die Hälfte des ursprünglichen Wortlautes wiedergewonnen sein, und zwar keineswegs am Stück, sondern oft in kleinen, kaum verwertbaren Bruchstücken. Vom ursprünglichen Umfang kann man sich dadurch eine recht gute Vorstellung machen, daß die vergleichsweise besterhaltene Handschrift, der pCarlsberg 313, mit Seitenzahlen versehen ist, deren höchste erhaltene immerhin 24 lautet, und zwar mit jeweils 30 langen Zeilen pro Seite.

Sämtliche bisher bezengten Bruchstücke lassen sich nach paläographischen Kriterien in die Römerzeit datieren. Allerdings deuten sprachliche und inhaltliche Kriterien auf eine frühere Entstehung. Wieviel früher, soll an dieser Stelle nicht ge-

\footnotetext{
${ }^{1}$ Sehr herzlich danken möchte ich an dieser Stelle der Deutschen Forschungsgemeinschaft, ohne deren Unterstützung durch Stipendien und Sachbeihilfen die langwierige und mit vielen Auslandsreisen verbundene Arbeit der Textaufnahme und Rekongtruktion nicht möglich gewesen wäre.

${ }^{2}$ Für Tebtynis wenigstens 17 verschiedene Handschriften innerhalb eines Zeitraumes won wohl allenfalls wenig über hundert Jahren.
} 
nauer diskutiert werden. Der Text ist ursprünglich mittelägyptisch abgefaßt worden, und diese Aufzeichnungsform ist in den konkreten Papyri auch die bei weitem häufigste. Allerdings gibt es auch einige Textzeugen einer demotischen Übersetzung. Dabei zeigen die meisten Handschriften jeweils nur eine Version. Lediglich eine schlecht erhaltene angeblich aus Elephantine dürfte biling gewesen sein, jedenfalls enthält sie vermutlich abwechselnd hieratisch und demotisch in jeweils sehr schmalen Kolumnen. Zumindest für einen Abschnitt des Textes ist auch eine Übersetzung ins Griechische nachzuweisen, für die es sogar zwei verschiedene heute erhaltene Papyri als Textzeugen gibt.

Im großen kann man folgende Einteilung des Textes feststellen: Am Anfang steht ein kurzer historischer Abschnitt. Ihm folgt die architektonische Sektion mit detaillierten Angaben über den Aufbau eines idealen ägyptischen Tempels sowie eine Liste der im Tempel zu verehrenden Götter. Weiter gibt es Angaben über Priester im allgemeinen sowie genaue Dienstpflichten sämtlicher Priester oder Tempelbediensteten.

Der Beginn des Textes ist derjenige Abschnitt, der bisher bereits am besten bekannt ist, da zwei Handschriften schon ganz bzw. großteils publiziert worden sind (und eine weitere, wenn auch unerkannt, bereits als Photographie veröffentlicht wurde). Zum einen ediert worden ist der pWien D 6319, den Reymond zusammen mit anderen Fragmenten unter dem Titel „From Ancient Egyptian Hermetic Writings ${ }^{\alpha}$ publiziert hat. ${ }^{3}$ Diese Handschrift stellt bis heute die besterhaltene Version der demotischen Übersetzung dar. ${ }^{4}$ Reymonds Übersetzung ist allerdings aufgrund paläographischer und philologischer Mängel sowie fehlerhafter Anordnung vieler Fragmente mit großer Vorsicht zu genießen. ${ }^{5}$ Zum anderen handelt es sich um den von G. Burkard bereits vollständig edierten pBerlin 23071 vs., ${ }^{6}$ der von mir bereits früher als direkt parallele mittelägyptische Version des von Reymond edierten Textes nachgewiesen worden ist. ${ }^{7}$ Da diese beiden Handschriften sowie eine Reihe wei-

${ }^{3}$ E. A. E. Reymond, From the Contents of the Libraries of the Suchos Temples in the Fayyum, Part II. From Ancient Egyptian Hermetic Writings, MPER NS 11 (Wien 1977).

* Nach Absonderung einiger nicht zugehöriger Bruchstücke, Anfügung einiger neuer sowie zahlreicher verbesserter Zusammensetzungen können alle Fragmente zu Resten von zwei direkt aufeinanderfolgenden stattlichen Seiten verbunden werden (Blatthöhe $\mathbf{c a} .35 \mathrm{~cm}$, Kolumnenbreite ca. $22,5 \mathrm{~cm}$ ).

${ }^{3}$ Für Nichtdemotisten dürften kurze Hinweise zu den sonstigen in dem betreffenden Buch edierten Texten hilfreich sein: Text B, der mit den von E. A. E. Reymond, op. cit. Part I. A Medical Book from Crocodilopolis. P. Vindob. D 6257, MPER NS X (Wien 1976) als Kolumne 1 und 2 von pWien D 6257 edierten Fragmenten zusammenpaßt, ist ein chemischer (bzw. protoalchemistischer) Traktat, der sich offenbar mit Textilfärberei beschäftigt. Text $G$ und $G_{1}$ sind eine Handschrift des „Thotbuches", s. dazu R. Jasnow, K.-Th. Zauzich, A Book of Thot?, in Chr. Eyre (Ed.), Proceedings of the Seventh International Congress of Egyptologists, Cambridge, 3-9 September 1995, OLA 82 (Leuven 1998), S. 607-618. Text D ist astrologischer Natur, s. G. R. Hughes, An Astrologer's Handbook in Demotic Egyptian, in: L. H. Lesko, Egyptological Studies in Honor of Richard A. Parker. Hannover/London 1986, S. 53-69 (bes. S. 69) und M. Smith, Lexicographical Notes on Demotic Texts II, Enchoria 13 (1985), S. 111-114.

' G. Burkard, Frühgeschichte und Römerzeit: P. Berlin 23071 vso., SAK 17 (1990), S. 107-133. Eine Kollation des Originals erbrachte einige verbesserte Lesungen.

${ }^{7}$ J. F. Quack, P. Wien D 6319. Eine demotische Übersetzung aus dem Mittelägyptischen, Enchoria 19/20 (1992/93), S. 124-129. 
terer unpublizierter Bruchstücke sämtlich mit demselben Passus am oberen Rand einer Seite einsetzten, besteht einige Wahrscheinlichkeit, daß es sich um den tatsächlichen Anfang der Textkomposition handelt und lediglich wenige Worte zu Beginn der ersten Zeile ganz vertoren sind.

Diese Einleitung des Gesamttextes ${ }^{8}$ führt weit in die Ceschichte Agyptens zurück; und zwar soll ein altes Dekret des Königs Neferkasokar zur Zeit des Cheops aufgefunden worden sein. Dieser recht obskure Herrscher gehört in die zweite Dynastie und war bisher fast ausschließlich durch seine Nennung in den Königalisten bekannt. Angeblich soll es damals eine siebenjährige Hungersnot gegeben haben, in deren Folge die Tempel in Ägypten verfielen. Nach dem Wiedereinsetzen der Nilüberschwenmung träumt der Pharao davon, daß er sämtliche Tempel in ganz Ägypten restaurieren lassen soll.

Das darüber abgefaßte Dekret wird später im Tempel von Heliopolis durch den Prinzen Hordjedef, den Sohn des Königs Cheops aufgefunden. ${ }^{9}$ Dieser läßt es nea abschreiben und mit dern Namen des Cheops versehen im Bücherhaus aufbewahren.

An diese historische Einleitung über die angebliche Entstehung und Wiederauffindung des Textes schließt sich eine programmatische Ubersehrift an, die als eigentlicher Titel des Werkes angesehen werden kann: „Vorschrift, die dem $13 y . t l s 3 b$, Wesir, obersten Vorlesepriester und königlichen Architekten, der die Arbeiten Pharaos im ganzen Land leitet, aufgetragen wurde bei der Grïndung jedes Tempels von Ober- und Unterägypten, um alle (Dinge) an ihren richtigen Platz im Tempel zu setzen. Vorschrift zum korrekten Verhalten, die jedermann aufgetragen wurde, um ihn in seine Arbeit in seinem Stundendienst in jedern Tempeldienst einzusetzen, um Reinheit festzusetzen und Tabus zu verabscheuen, sich an die Vorschriften des ersten Males zu halten durch jeden Zugangsberechtigten des Tempels, beginnend mit den Oberpriestern, die beim Gott eintreten bis zu jedem jeweiligen Amt.

Aus dieser Uberschrift läßt sich recht deutlich die doppelte Zielrichtung des Werkes erkennen. Zum einen wird die architektonische Anlage des Tempels vorgeschrieben, zum anderen finden sich Pflichten der Priester, speziell Dienstpflichten für jeden einzelnen, der im Tempel beschäftigt ist. Ein wichtiger Punkt, der betont werden muß, ist ferner die Allgemeingültigkeit der Vorschriften. Das im Buch vom Tempel entworfene Bild ist der Intention nach nicht die Beschreibung eines konkroten Tempels, sondern ein Idealbild, das nach Möglichkeit bei der konkreten Anlage jedes einzehen Tempels von Ober- und Unterägypten verwirklicht werden soll.

${ }^{8}$ Eine detaillierte Behandlung dieses Abschnittes bei J. F. Quack, Der historische Abschnitt des Buches vom Tempel, in: E. Blumenthal, J. Assmann (Hrsg.), Literatur und Politik im pharaonischen und ptolemäischen Ägypten (Kairo 1999), i. Dr. Enst nach Abschluß aller Korrekturen für das betreffende Manuskript habe ich bemerkt, daß es noch einen weiteren, bereits publizierten Textzeugen gibt. Auf dem von M. Manfredi, O. Neugebauer, Greek Planetary Tables from the Time of Claudius, ZPE 11 (1973), S. 101-114, T. III veröffentlichten Text gibt es in Frg. 9 eine, in Verhältmis zum Griechischen auf dem Kopf stehiende hieratische Zeile, die in der Publikation noch als unklar eingestuft wurde, soweit das Photo lesbar ist, jedoch zweifelsfrei die enste Zeile des Buches vom Tempel darstellt, die offenbar als Schreibübung verwendet wurde.

" Dieser Prinz gilt auch sonst als Entdecker alter Schriften, besonders wird ihm die Auffindung der Totenbuchkapitel 130 sowie 64 (Langfassung) + 30B zugesehrieben; für eine detaillierte Argumentation zu letzterem Fall, der die übliche, aber sachlich ungenane Angabe Tb $30 \mathrm{~B}, \mathrm{~Tb} 64$ und Tb 148 ablösen soll, muß ich auf den Kommentar in meiner Edítion verweisen. 
Am Anfang des Bauabschnittes steht die Gründungszeremonie. Sie findet als feierliche Zeremonie in Form des traditionellen Strickespannens durch den König und die Göttin Seschat statt. Der gesamte Tempel wird anschließend von innen nach außen beschrieben. Die Anfangspassagen sind leider schlecht erhalten. Offenbar wird die Fundamentgrube ausgehoben; um den zentralen Schrein herum soll es rechts vier, links fünf weitere Schreine geben. Ferner sollen Krypten gebaut werden, in denen primär die unterirdischen Götter des betreffenden Gaues sowie die unterirdischen Götter, die in keinem Gau fehlen, ruhen sollen. Im Fall von Unruhen sollen hier sämtliche Götter ${ }^{10}$ untergebracht werden. ${ }^{11}$ Jeweils rechts und links eine Treppe sollen wohl za diesen Krypten hinabführen, aber nur bei Festen zugänglich sein.

Offenbar sollen im inneren Bereich auch Schreine für die große und kleine Götterneunheit sowie weitere Götter von Ober- und Unterägypten untergebracht werden. Hier befinden sich auch zwei Räume praktischer Nutzung, nämlich die Kleiderkammer ( $h w . t-m n h . t)$ und die Speiseopferkammer ( $h w . t-b w-\breve{c} f 3 . w)$.

Eine große Halle mit Säulen schließt sich an; vermutlich als Zentralhalle (wsh.t $h r(t-l b)$ bezeichnet. In diesem Bereich, dessen Beschreibung leider sehr schlecht erhalten ist, finden sich noch eine Reihe weiterer Kapellen und Hallen. Dabei wird eine Zugangsrestriktion aufgestellt, die nur Propheten (und eventuell weiteren Ämtern) den Zugang erlaubt.

Es folgt die Opferhalle (wsh.t htp.w). Etwa in diesem Bereich sollen zwei weitere Treppenhäuser gebaut werden, die diesmal nach oben führen. Sie leiten zu einer Beschreibung von Dachkapellen über, die vor allem beim Neujahrsfest eine Rolle spielen sollen. Ein als Pronaos ( $h n t l)$ bezeichneter großer Gang mit Säulen schließt diesen ganzen Tempelbereich ab. Er soll in seinen Dimensionen Breite und Höhe über den bisherigen Bau hinausgehen. Der Kernbereich wird von einem Umgang ungeben. Etwa in diesen Bereich sollen auch Statuen der Könige von Ober- und Unterägypten kommen, wobei sie ihr Gesicht dem Tempel zuwenden. ${ }^{12}$ Innerhalb des Pronaos gibt es zwei Kammern für Gerätschaften. Dieser innere Tempelbereich soll nur für sogenannte "hochrangige Priester der Gottesrituale ${ }^{4}$ zugänglich sein.

An dieser Stelle erfolgt nochmals eine Bauvorschrift für Kapellen auf dem Dach, die diesmal speziell als Festplatz des ersten Festes ( $s . t h 3 b$ tpl) bezeichnet werden. Die andersartige Terminologie deutet darauf hin, daß es sich nicht um ein reines Duplikat zu vorherigen Erwähnungen von Bauten auf dem Dach handelt, sondern um eine andere Örtlichkeit. In diesem Bereich sollen die himmlischen Götter des jeweiligen Gaues sowie diejenigen himmlischen Götter, die in keinem Gau fehlen, un-

\footnotetext{
${ }^{10}$ Mit ${ }_{n}$ Cöttern ${ }^{\mu}$ sind hier ganz real die kostbaren Statuen gemeint; man vgl. etwa die bisher

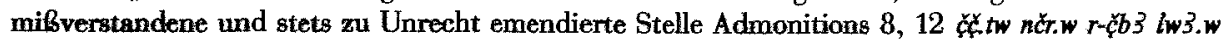
„Cötter(bilder) werden für Rinder verkauft" (Editionen A. H. Gardiner, The Admonitions of an Egyptian Sage from a Hieratic Papyrus in Leiden (Pap. Leiden 344 Recto) (Leipzig 1909), S. 65; W. Helck, Die „Admonitions“. Pap. Leiden I 344 recto, KÄT 11 (Wiesbaden 1995), S. 40.

"Diese Angaben zeigen sehr schön die Doppelfunktion der Krypten, die zum einen durchaus echte Kulträume sind, zum anderen jedoch auch eine ausgeprägte Schutz- und Sicherungsfunktion haben. S. hierzu etwa W. Waitkus, Die Texte in den unteren Krypten des Hathortempels von Dendera. Thre Aussagen zur Funktion und Bedeutung dieser Räume, MÄS 47 (Mainz 1997).

12 Es ist mir nicht völlig klar, ob sich diese Angabe auf fest im Tempel aufgestellte Statuen bezieht oder auf tragbare Statuen, die bei Prozessionen mitgeführt werden. Letzteres dürfte angesichts des Verbs iwi nkommen ${ }^{4}$ plausibler sein.
} 
tergebracht werden. Zwischen zwei Kapellen soll speziell ein Kiosk mit Säulen gebaut werden, der oberhalb eines Schreines (vermutlich des zentralen Sanktuars) im Erdgeschoß gebaut werden soll. Dort soll sich an einer aufgrund von Lïcken im Text nicht ganz deutlichen Stelle zum einen das Goldhaus, zum anderen der Bezirk der heiligen Tiere ( $m 3{ }^{2} r w$, im Demotischen übersetzt als ${ }^{2}{ }^{2}$ ' $\left.w \cdot t-n c r n\right)$ befinden. Dabei wird betont, daß die Tiere sich wohl füblen, wenn sie die Sonne sehen.

Der folgende, sehr schlecht erhaltene Bereich scheint sich mit dem Litaneiensasl (wsh.t wtn.w) zu beschäftigen. Wo der Zusemmenhang wieder klarer wird, erhält man die Aufzählung der Schreine, die sich rechts und links in ihm befinden sollen. Zuerst genannt wird auf der rechten Seite das bekannte, Morgenhaus" ( $p r-f w 3)$, in dem die Reinigung des Königs beim Eintritt in den Tempel stattfindet. ${ }^{13}$ Daneben befindet sich das Bücherhaus ( $p r$-mç3.t), anschließend ein davon unterschiedenes „Schriftenhaus ${ }^{\star}\left(p r-s h 3^{2}\right)$, zuletzt der Platz der Türhüter (s.t n.t lriw-3).

Auf der linken Seite befinden sich das Natronhaus (hw.t-hsmn), der Platz der Kranzmacher (s.t iri.w-mhw), ein schlecht erhaltenes Gebäude, der Platz des Sakristans(?) (s.t $\left.k 3^{3} w . t i^{\prime}\right)$, ${ }^{14}$ der Platz der Opferbringer (s.t msi-wth.w). Nach vorme wird dieser Bereich durch Pylontürme abgesehlossen. Die Umfassungsmauer in diesem Bereich, deren Breite (8Ellen) und Höhe (nicht erhalten) ausnahmsweise genau festgelegt sind, wird mit einer vorgeschriebenen $Z a h l$ von Türen versehen. Die schlechte Erhaltung macht die Beurteilung schwierig, doch scheinen es einschließlich des Haupteingangs zwischen den Pylonen nur drei (allenfalls fünf) Türen zu sein.

An diesen Bereich schließt sich ein großer Raum an, der offenbar in aeinen Dimensionen über das bisher Beschriebene hinausgehen soll. Er wird als ${ }_{n}$ Hof der Menge (wst.t ms') bezeichnet. Auch in ihm gibt es einen Ort zur Reinigung des Pharao. Noch zwei weitere Höfe, jeweils mit Pylonen schließen sich an, wobei der innere davon der Standort der [Frauen(?)] sein soll. Insgesamt ergeben sich damit mutmaßlich vier verschiedene Pylone. Eine so hohe $\mathrm{Zahl}$ ist vorwiegend aus Bauten des Neuen Reiches, z. B. dem Tempel von Karnak bekannt, während Tempel der griechisch-römischen Zeit meist nur einen oder allenfalls, wie der Tempel von Philä, zwei Pylone aufweisen.

Anschließend werden die Räumalichkeiten aufgezählt, die sich außerhalb neben der Mauer befinden. Genannt werden u. a. das Goldhaus zurn Herstellen der Götterstatuen und zum GieBen der Kultgeräte, der Schlachthof (shw-hr $n$ stp.w), die Salbenküiche (s.t $n$ iri $\left.m c^{x}, t\right)$ und anderes, darunter vermutlich auch die Latrine (s.t $\left.n . t s^{* t}\right)$. Hier soll sich auch ein See befinden. Eine Reihe von Priestern und Tempelbedienstëten haben hier ihre ${ }_{n}$ Plätze ${ }^{\star}, \mathrm{d} . \mathrm{h}$. wohl Wohnquartiere für die Zeit ihres Tempeldienstes. Genannt werden u. a. der Astronom, der Barbiex, der Schulmeister, der Gesanglehrer, der Färber, der Arzt. Hier soll es insgesamt fünf Türen geben, einschließlich der großen Tür in der Hauptachse. Die Umfassungsmauer soll zwanzig Ellen hoch sein, ihre Dicke ist bisher nirgends erhalten.

Im Umfeld des Tempels außerhalb dieser Mauer befinden sich weitere Einrichtungen praktischer Natur. Viehhürde und Geflügelhof haben ebenso ihren Platz wie

${ }^{13}$ A. M. Blackman, The House of Morning, JEA 5 (1918), S. 148-165.

${ }_{14} \mathrm{Zu}$ diesem Titel s. u. a. die Bemerkungen von J. Leclant, Enquêtes sur les sacerdoces et les senctuaires égyptiens à l'epoque dite néthiopienne (XXV* dynastie), BdE 17 (Kairo 1954), S. 68 und G. Vittmann, Priester und Beamte im Theben der Spätzeit (Wien 1978), S. 120 u. 129-131. Die meist übliche Deutung als ,Träger ${ }^{\alpha}$ dürfte kaum aufrecht zu halten sein. 
Getreidespeicher und Stätten der Lebensmittelverarbeitung. In einer Ecke befindet sich eine Latrine. Auch die Balsamierungsstätte ( $w^{\prime} b . t$ ) der heiligen Tiere soll bier gebaut werden. Sie wird in ihrer räumlichen Anlage etwas eingehender beschrieben. Offenbar gibt es drei Schreine, von denen zwei als Resenet und Mehenet, also die traditionellen Herstellungsorte für Mumienbinden bezeichnet werdon, sowie eine zentrale Halle.

In der Folge erwähnt werden Speicher für verschiedene Nutzobjekte, so Textilien und Matten, Stricke, Zeltstangen, Holzkohlen für das Altarfeuer Auch Töpferei (s.t $n . t\left\langle t a t s\right.$ ) und Abfallhaufen (s.t. n.t $\left.b 3^{\circ} b w . t\right)$ dürfen nicht fehlen. Das gesamte Geviert der Tempelanlage soll mit einer zwölf Ellen dicken und zwanzig Ellen hohen Mauer umschlossen werden. Erneut werden die Türen in ihren vorgeschriebenen Plazierungen angegeben.

Nach einigen schlecht erhaltenen Sätzen erhält man eine Beschreibung des Geburtshauses (pr-msi.t). Von den Angaben ist noch erkennbar, dab es eine Festhalle hatte und daß auf einer Säule eine Dekoration aus Tanzzwergen (hyt) angebracht sein soll.

Im nach hinten hin immer schlechter erhaltenen Bauabschnitt ist anschließend noch erkennbax, daß u. a. die Frage von Seen und Reinigung eine Rolle spielt, auBerdem wird ein Festhous ( $h w . t-h^{\circ} i w$ ) erwähnt. ${ }^{15}$ Isoliert und daher schlecht auswertbar ist die Angabe, daß, sofern es sich um den Tempel einer männlichen Gottheit handelt, Palmen- und Papyrussäulen rechts und links [der Mittelachse(?)] stehen sollen. Man erfährt noch von einem See, der mit Lotus bewachsen sein soll, von einem Damm sowie einem Kanal und einer Landestelle mit Kai ( $\breve{c} \zeta \xi \xi)$.

Offenbar geht die Beschreibung jetzt zum heiligen See und dem heiligen Hügel über, für die besonders scharfe Zugangarestriktionen herrschen. Zugelassen scheinen Sängerinnen sowie hochrangige Priester Nach einer nicht sicher zu fïllenden Lücke setzen die Handschriften wieder ein. Man erfährt, daß Personen, die ohne Autorisation ertappt werden, wie sie in den Bereich des heiligen Sees eindringen, so behandelt werden sollen wie jemand, der den Namen des Herrschers lästert, d. h. sie sollen getötet und auf den Feueraltar gelegt werden. ${ }^{16}$

In diesem Bereich spielen naturgemäß die Osirisrituale und die damit verbundene Herstellung von Figurinen eine große Rolle. Man erfährt davon, wie das „un-

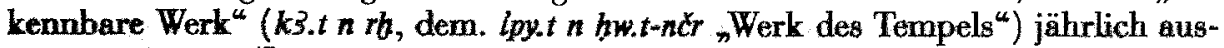
gewechselt wird. ${ }^{17}$ Religionsgeschichtlich nicht ganz uninteressant dürfte sein, daßs dieses Auswechseln (lqn) im Demotischen als Verjüngen ( diesem Bereich dargebrachte Opfergaben sollen anscheinend nicht verzehrt, sondern in den Fluß geworfen werden.

Hier erreicht man, nach etwa sieben bis acht Seiten der verschiedenen Handschriften, einen größeren Einschnitt, der das Ende der reinen Architekturbeschreibung bedeutet. Es folgt, sozusagen als Anhang dazu, die Liste der im Tempel zu verehrenden Götter. Während die architektonische Sektion ungeachtet größerer Lücken im ganzen in eine gesicherte Abfolge gebracht werden konnte, stellt die

15 Eventuell handelt es sich dabei um eine Stationskapelle für Götterprozessionen.

16 Vgl. A. Leahy, Death by Fire in Ancient Egypt, JESHO 27 (1984), S. 199-206.

77 Zum Zyklus der Herstellung und der Deponierung der Choiakfigurinen, um die es hier geht, s. grundlegend E. Chassinat, Le mystère d'Osiris au mois de Khoiak (Kairo 1966-68), in neuerer Zeit etwa Chr. Leitz, Die obere und die untere Duat, ZÄS 116 (1989), S. 41-58. 
Götterliste ein größeres Problem dar Dies beginnt bereits auf der rein praktischen Ebene damit, daß Fragmente mit Resten einer Architekturbeschreibung grundsätzlich hochverdächtig sind, zum Buch vom Tempel zugehören, während Reste von Götterlisten prinzipiell auch zu ganz anderen Kompositionen, z. B. Opferlitaneien eines Rituals gehören können. Die Existenz dieser Sektion konnte folglich erst etabliert werden, als mehrfach und von mehreren verschiedenen Handschriften Fragmente von Götterlisten denselben Schriftduktus aufwiesen wie Papyri, die unzweifelhaft zum Buch vom Tempel gehören, und schließlich sogar der direkte Übergang von der Banbeschreibung zar Götterliste auftauchte.

Die Götterliste muß, da sie Allgemeingültigkeit beansprucht, eine Reihe von Freiräumen für individuelle Gestaltung haben. Selbstverständlich ist dies bei der Angabe des Tempelherrn, der leider nur unsicher und unvollständig erhalten ist, vermutlich hieß es , der große Gott am [Uranfang]. "Sonst findet sich etwa die Angabe „die unterirdischen Götter des betreffenden Gaues und die unterirdischen Götter, die in keinem Gau fehlen. "Die Liste ist nach Räumlichkeiten organisiert, in der Praxis mußs allerdings die Lokalisierung anhand erhaltener Angaben Hand in Hand gehen mit einer Theoriebildung anhand der erwähnten Gottheiten. Hier sollen nur einige relativ klare Punkte erwähnt werden.

Im Bereich der Opferhalle finden sich u. a. die sieben heiligen Kühe und der Stier, die aus Tb 148 als Nahrúngsspender bekannt sind, aber auch vielfach tatsächlich in Tempeln erscheinen ${ }^{18}$ die stiergestaltigen „Herren der Altäre" Apis, Sema-Wer, Mnevis und Ageb-Wer, ${ }^{19}$ die Wahrnehmungsgötter Hu, Sia, Iru und Sedjen sowie die nahrungspendenden göttlichen Kühe Sechat-Hor, Schedit, Hesat und Werit.

Nicht von den Textangaben her lokalisierbar, aber durch die auftretenden Götter recht gut zu plazieren ist ein weiterer Abschnitt, der in mehreren Textzeugen erhalten ist. In ihm erscheinen nämlich die charakteristischen Genien des Zyklus von Sonne und Mond, die in letzter Zeit Gegenstand eingehender Untersuchungen waren $^{20}$ und in ähnlicher Form auch in einer Kodifikation priesterlichen Wissens (pBerlin 7809, 3,14-4,10) erscheinen. ${ }^{21}$ Tatsächlich ist eine Handschrift, die als Parallele zu einer solchen onomastischen Liste publiziert wurde ( $\mathrm{pBerlin} 14460),{ }^{22}$ in Wirklichkeit dem Buch vom Tempel zuzuweisen. ${ }^{23}$

Das Ende der Götterliste verliert sich im Dunkeln, wie überhaupt von hier an meist isolierte Textbruchstücke mehr oder weniger guter Erhaltung dominieren, von denen einige nach Inhalt oder erhaltenen Seitenzahlen recht genau plaziert

${ }^{18}$ A. v. Lieven, Book of the Dead, Book of the Living, BD Spells as Temple Texts, in: S. Seidlmayer (Ed.), Religion in Context. Imaginary Concepts and Social Reality (i. Dr.).

${ }^{19} \mathrm{Zu}$ diesen Göttern s. J.-L. Simonet, Le collège des dieux maîtres d'autel (Montpellier 1994).

${ }^{20}$ F. Labrique, Les escortes de la lune dans le complexe lunaire de Khonsou à Kamak, BSFE 140 (1997), S. 13-26; dies., L'escorte de la lune sur la porte d'Éuergète à Karnak, I. Les documents, in: R. Gundlach, M. Rochholz (Hrsg.), Vierte ägyptologische Tempeltagung. Feste im Tempel, ÄAT 33/2 (Wiesbaden 1998), S. 107-149. Weitere Bemerkungen bringt A. v. Lieven, Der Himmel über Esna. Eine Fallstudie zur Religiösen Astronomie (i. Dr).

21 J. Osing, The Carlsberg Papyri 2. Hiexatische Papyri aus Tebtunis I (Kopenhagen 1998), S. $288-290$, T. 29.

${ }^{22}$ Osing, Hieratische Papyri aus Tebtunis I, S. 297, T. 30.

${ }^{23}$ Das Fragment, an das ich inzwischen nach zusätzliche Bruchstücke anschließen konnte, gehört dem Duktus nach sicher zum pCarlsberg 383. 
werden können, während andere völlig haltlos herumtreiben. Der große Übergang vom Tempel als Bau und als Ort der Götter zu den in diesem Bereich agierenden Personen, nämlich den Priestern und Tempelbediensteten bleibt somit völlig offen.

Am Anfang des Abschnittes über das Tempelpersonal stehen eine Reihe grundsätzlicher Bestimmungen. Zuerst erwähnen möchte ich einen Abschnitt, der im ägyptischen Text relativ schlecht erhalten, aufgrund seines Inhaltes jedoch von erheblicher Bedeutung ist. ${ }^{24}$ Dabei handelt es sich um Reste eines Eides, den Priester vermutlich bei ihrer Weihe sprechen müssen und der bereits aus zwei griechischen Papyri, nämlich dem $\mathrm{pWashington} \mathrm{University} \mathrm{inv.} 138 \mathrm{r}$. und dem pOslo $2 \mathrm{r}$. bekannt wax. Genauer gesagt sollte man allerdings von mindestens zwei verschiedenen Eiden sprechen. Zu Anfang des griechischen Textes ist zunächst noch von Fragen der Reinheit die Rede, danach erst beginnt im pWashington University inv 138 , I, Z. 17, mit 8̄ eingeleitet, in wörtlicher Rede der Text des Eides. Diesem Einschnitt entspricht exakt der Befund des hieratischen Fragmentes, das direkt vor den Satz ${ }_{n}$ ich werde kein Tabu essen “ die Einleitung der wörtlichen Rede $(\not t+m \neq w)$ setzt. ${ }^{25}$ Im griechischen Text pOslo $2 r^{26} \mathrm{Z} .3-11$ ist der Einschnitt zwischen den beiden Eiden teilweise erhalten, es wird betont, daß auch die Verwandten zugegen sein sollen, dann folgt der zweite Eid, der zu schwören ist, wenn die betreffende Person in den Rat berufen wird. Auch in demjenigen hieratischen Fragment, das teilweise Text enthält, der dem pWashington University inv. 138, Kolưnne II entspricht, stehen zu Anfang einige schlecht erhaltene Zeilen, die unter anderem davon sprechen, jernand sollte den Gott anbeten, was vermutlich zur Einleitung des dann folgenden Eides gehört.

Hier soll ein Versuch gemacht werden, vom hieratischen Text ausgehend den Wortlaut der Eide zu rekonstruieren, wobei der griechische Text nur mit Vorsicht zur Ergänzung herangezogen wurde, da er vermutlich gegenüber dem ägyptischen Vorbild erheblich gekürzt ist: ${ }^{27}$

[.... beim Fest des Viertelmondes(?), beim [......] ihn einführen; ihn den Gott [anbeten] lassen [......] mit den Worten: „Ich werde nicht irgendein Tabu eines [Priesters] essen [......, ...] sie beim Gott, [ich] werde nicht meinen Arm [aus-

${ }^{24}$ Zu diesem Abschnitt s. J. F. Quack, Ein ägyptisches Handbuch des Tempels und seine griechische Übersetzung, ZPE 119 (1997), S. 297-300. Nachzutragen ist dort die Neuedition der einen griechischen Handschrift bei K. Maresch, Z. Packman, Papyri from the Washington University Collection, St. Louis, Missouri, Part II, Pap. Col. XVIII (Opladen 1990), S. 31-35 als Nr. 71. Zu durch die Isismysterien vermittelte Echos in der römischen Literatur s. L. Koenen, Priestereid und römische Elegie, ZPE 2 (1968), 31-38; ders., Egyptian Influence in Tibullus, Illinois Classical Studies 1 (1976) 127-159, bes. 128f,; O. Raith, Unschuldsbeteuerungen und Sündenbekenntnis im Gebet des Enkolp an Priap (Petr. 133.3), Studii Classici 13 (1971), 109-125; R. Merkelbach, Unschuldserklärungen und Beichten, in ders., Philologica. Ausg. Kleine Schriften, Stuttgart und Leipzig (1997) 95-112.

${ }^{25}$ Dieser Befund ergab sich erst nach der Veröffentlichung des Aufsatzes in der ZPE 119, als ich im Frühling 1999 zwei kleine weitere Bruchstücke in Florenz identifizieren und anpassen konnte.

${ }^{26}$ Durch den Befund des hieratischen Textes dürfte es definitiv gesichert sein, daß der Text des Osloer Papyrus, vermutlich mit geringer Überlappung, weitgehend zwischen die erhaltenen Teile der ersten und zweiten Kolumne der Washingtoner Handschrift gehört.

27 Das ZPE 119, S. 297 u. 298 Anm. 15 erwähnte Fragment des pCarlsberg 312 ist versuchsweise an den Anfang des Abschnittes gestellt worden. 
strecken], um einen Kopf abschlagen [..... ich] werde(?) keinen Mann töten, [...... $]$., ich habe niemals [......, ich werde mich nicht verei]nen [mit .....] in Unreinheit $[. . . . .$.$] ihre [. ..] \mathrm{u}[$ nd ....... ....... Veranlassen, daß $]$ er den Gott anbetet bei der. Treppe [......] im Tempel [.....] ausgenommen [...] der beiden Länder Nicht [...... . im Rat des Tempels ... nicht [...... ihn alle Menschen in [...] des Tempels [......] für ihn auf ihr. Ich esse nicht und trinke nicht, [was ein Tabu ist ...... in den] Schriften, ich komme ihnen mit meinen Fingern nicht nahe. [......] auf [der Tenne.] Ich werde keine [Waage] hochheben [...... Ich werde kein] Feld [vermessen .......]. ${ }^{\star}$

Ein relativ großer in seiner Abfolg gesicherter Bereich behandelt zunächst die Frage der materiellen Versorgung. Angesichts der Tatsache, daß dabei auch die Einweihung beim Gott erwähnt wird, besteht einige Wahrscheinlichkeit, daß dieser Abschnitt dicht auf den eben genannten folgt. Die Einzelheiten bleiben leider oft im Dunkeln, erkennbar ist aber, daß materielle Besitztümer zugewiesen werden. Auch an die Versorgung Hinterbliebener wird gedacht. So soll etwas (vermutlich Einkünfte) an die Kinder gegeben werden, damit sie davon leben, und zwar, wie ausdrücklich betont wird, die weiblichen ebenso wie die männlichen Kinder Die Witwe wird im Zweifelsfall ebenfalls versorgt, soll aber offenbar nichts aus dem Schatzhaus erhalten. Auch Lebensmittel, Fette, Öle und Aromastoffe werden in diesem Bereich erwähnt. In einem leider aufgrund schlechter Erhaltung nicht klaren Fall soll wiederum Besitz an die Kinder gegeben werden, die Hälfte jedoch an einen Gehilfen. Eventuell wird hier im Falle sehr kleiner Kinder zunächst ein Vertreter eingesetzt, der den tatsächlichen Tempeldienst für eine Reihe von Jahren übernimmt.

Anschließend erfolgt eine Auflistung desjenigen Personals (smt.t. $)$, daß aufgrund körperlicher und charakterlicher Mängel nicht beim Gott eingeweiht wird. Genannt werden körperliche Abnormität, etwa in der Körpergröße, Verstümmlungen an den Gliedern, weiße Färbung (Albinismus), rote Färbung ${ }^{28}$ und ähnliches. Unter den Charaktermängeln finden sich unter anderem übermäßiger Genuß von Wein und Bier, Lügen, Verbreiten von Gerüchten, Habgier, Großmäuligkeit, Auflehnung gegen Vorgesetzte, Diebstahl und unzüchtiges Verhalten. Die betreffenden Leute werden angesehen als solche, die ${ }_{n}$ in der Gestalt des Seth, des Apopis und jenes Gottes sind, gegen den in Memphis der Arm ausgestreckt wird." Während die beiden ersteren als Negativgestalten in Ägypten gut bekannt sind, bleibt der letzte einstweilen im Duakeln.

Nach einem schlechter erhaltenen Bereich, der nochmals Fragen der Weihe behandelt, werden für einige Bereiche des Tempels diejenigen Priesterränge aufgezählt, die allein dort Zutritt haben. Hier ein etwas längeres Zitat: „Was jeden heiligen Hügel und [jeden heiligen] See betrifft, der im Inneren des heiligen Hügels ist, so tritt dort niemand ein, ausgenommen die hochrangigen Priester (wb.w $3 . w)$ und die Zugangsberechtigten ( $\left.k, w /{ }^{\prime \prime} k 3 . w\right)$. [Was] den heiligen Hügel [betrifft], der sich

${ }^{28}$ Die Ägypter hatten gegenüber rothaarigen Personen stets ein großes Mißtrauen, sie wurden teilweise registriert, nach Angaben griechischer Schriftateller sogar als Menschenopfer dargebracht. S. etwa E. Edel, Das Akazienhaus und seine Rolle in den Begräbnissen des alten Ägypten, MÄS 24 (Berlin 1970), S. 39-41; J. Yoyotte, Héra d'Héliopolis et le sacrifice humain, Annuaire EPHE, $V^{\top}$ section - sciences religieuses 89 (1980-81), S. 31-102. 
in jeglichem Gau von Ober- und Unterägypten befindet, so tritt kein Priester in ihn ein, außer dem Ritualleiter ( $h r l-s_{s}^{\zeta}+3$ ), vier Mann, und dem 'b.w-nčr; acht Mann, Summe zwölf. Man setzt einen Ritualleiter und einen 'b.w-nčr in ihrem Monatsdienst zusammen mit den Zugangsberechtigten, die ihren Dienst verrichten, im heiligen See ein, der im betreffenden Hügel ist, entsprechend ihren Ritualen. Sie sind es, die zu jedem heiligen Hügel eintreten sollen und zu jedem heiligen See, der sich im Inneren des heiligen Hügels befindet, um dort Tag für Tag nach den Dingen zu fragen, um die Festrollen zu rezitieren mit den Sprüchen der Gottesrituale bei allen Festen des Himmels und der Erde, um zu reinigen mit Weihrauch und Wassergüssen." Im weiteren Verlauf geht es dann um die Arbeit an den Osirisfigurinen und die an ihnen vollzogenen Rituale, wobei wieder die zyklische Emeuerung beschrieben wird, indem man jeweils die Figurine des Vorjahres fortnimmt und entweder im heiligen Hügel beerdigt oder aber in den heiligen See oder am Flußufer ins Wasser wirft. Beschrieben wird auch, wie bei bestimmten Festen der Ritualleiter allein eintreten darf, nachdem seine Gefolgsleute zurückgehalten wurden, und auch er nur mit rückwärts gewandtem Kopf.

Nach einigen weiteren Sätzen, in denen u. a. die Nachfolgeregelung im Todesfall behandelt wird, endet die Textkontinuität wieder. Möglicherweise hierher gehörig sind Fragmente einer relativ schwer lesbaren Berliner Handschrift, in denen bestimmte Priestertitel, z. B. der Sem-Priester, definiert und mit göttlichen Konstellationen verbunden werden. Vor diesem Bereich wird in leider schlechter Erhaltung über die Auffindung einer Schrift berichtet.

Offenbar als Abschluß dieses Bereiches bzw. als Übergang zum nächsten Teil scheint noch einmal eine Fundgeschichte gebracht zu werden, von der allerdings wenig erhalten ist. Die Namen von König Cheops und Prinz Hordjedef sind jedoch beide vollständig erhalten.

Damit beginnt der letzte klar faßbare Bereich des Textes, der schätzungsweise fast die Hälfte des Gesamtumfangs ausmacht, nämlich die detaillierte Aufzählung des Tempelpersonals und ihrer Dienstpflichten. Diese Angaben sind in hohem Maße normiert und einheitlich stilisiert. Am Anfang steht jeweils die Angabe des jeweiligen Titels, es folgt die Personenzahl. Dabei ist klar erkennbar, daß mit einem System von rotierenden Phylen operiert wird, die sich in monatlichem Turnus abwechseln, wie es auch sonst als Praxis ägyptischer Tempel gut bekannt ist. Erwähnt werden sollte allerdings, daß grundsätzlich mit einem Bestand von vier Phylen operiert wird. Bekanntlich wurde durch das Dekret von Kanopus (237 v. Chr.) die Einrichtung einer fünften Phyle festgelegt. ${ }^{29}$ Das zur Zeit der Entstehung der

2 Hieroglyphischer und griechischer Text bei K. Sethe, Hieroglyphische Urkunden der griechisch-römischen Zeit. Urkunden II (Leipzig 1904-16), S. 140-154; alle drei Versionen bearbeitet bei W. Spiegelberg, Der demotische Text der Priesterdekrete von Kanopus and Memphis (Rosettana) mit hieroglyphischen und griechischen Fassungen und deutscher Übersetzung nebst demotischem Glossar (Heidelberg 1922); zur demotischen Version s. die neueste vollständige Übersetzung bei R.S. Simpson, Demotic Grammar in the Ptolemaic Sacerdotal Decrees (Oxford 1996), S. 224-241; zur griechischen Version 8. A. Bernand, Le Delta égyptien d'après les texts grecs, III (Cairo 1970), S. 989-1037. Zu den fünf Phylen der griechisch-römischen Zeit in konkreten Belegen s. außer Dittenberger (OGIS 56) und Bernand auch W. Otto, Priester und Tempel im Hellenistischen Ägypten. Ein Beitrag zur Kulturgeschichte des Hellenismus (Leipzig/Berlin 1905-08), S. 23-38. 
konkret erhaltenen Handschriften gültige System widerspricht also dem in den Texten beschriebenen. Darin kann man einen Hinweis sehen, daß der zugrundeliegende Text auf einen älteren Archetyp zurückgeht. Andererseits dürfte dieser Befund auch das Alte Reich als Entstehungszeit ausschließen, ds im Alten Reich ein kompliziertes System angewandt wurde, bei dem sich fünf Phylen mit jeweils zwei Unterabteilungen abwechselten. ${ }^{30}$. Einige wenige Ämter unterliegen nicht der Phylenrotation, sondern werden allem Anschein nach pernanent ausgeübt. Für diese Ränge gibt es üblicherweise gerade zwei Vertreter, die in der Benennung spezifisch unterschieden werden. Die Beschreibungen gehen, soweit sich die Stücke durch direkte Abfolge oder erhaltene Seitenzahlen ordnen lassen, hierarchisch von oben nach unten vor, beginnen also mit der obersten Leitung des Tempels bis hin zu den niedersten Arbeitern.

Die Dienstpflichten selbst sind fast durchgängig in der Form partixipialer Feststellungen gehalten, also „er ist es, der ... tut. “ Derartige Sätze folgen meist in längeren Ketten aufeinander. Thre Verwendung verbindet die vorliegende Komposition in besonderer Weise mit einem gut bekannten ägyptischen Text, nämlich den Dienstpflichten des Wesirs, ${ }^{31}$ und zwar inmerhalb jenes nicht ganz einheitlichen Textes vor allem mit dem dritten und letzten Abschnitt, der sich durch weitgehende Vermeidung des bestimmten Artikels als sprachlich besonders alt erweist. ${ }^{32}$

Gelegentlich finden sich Hinweise auf die Rangstellung, vor allem die Angabe, daß ein bestimmter Priester ,einer ist zusammen mit den hochrangigen Priestern", d. h. wohl, ihnen im Rang gleichwertig ist, bzw. zu ihnen zugehört. Am Schluß des Textes findet sich eine Nachfolgeregelung, die standardmäßig die Übergabe des Amtes vom Vater auf den Sohn vorsieht. In manchen Fällen wird noch angegeben, daß der Betreffende nach dem Dahinscheiden für sieben Dekaden in die Balsamierungswerkstatt gebracht werden soll. ${ }^{33}$

An erster Stelle steht der Gouverneur und Vorsteher der Propheten (h3.tl-'.w iml-r' $h m . w-n \nless r$ ), der gleichsam die Schnittstelle zwischen der staatlichen Zivilverwaltung und der inneren Tempeladministration derstellt. Er ist gerade einer der wenigen Ränge, die nicht der Rotation unterworfen sind. Dafür werden zwei verschiedene Stufen etabliert, nämlich der große Couverneur $(h 3 . t i-w w r)$ und der nachfolgende Gouverneur ( $h 3 t i-\mathfrak{w}$ lml-ht). Letzterer soll die abgelegten Gewänder des ersteren auftragen, für das Ruderfest wird speziell ein Pantherfell als Bekleidung angegeben. Bemerkenswerterweise wird angegeben, daß sie bei allen Festen den Apisstier einführen sollen. Darin kann man einen Hinweis darauf sehen, daß der Text ungeachtet seiner gesamtägyptischen Intention letztlich doch den Zustand der memphitischen Residenz zum Idealbild für alle Tempel macht. Generell sind es bei Festen

${ }^{30}$ Zum Phylensystem des alten Reiches s. P. Posener-Krieger, Les archives du temple funéraire de Néferirkarê-Kakaï (Les papyrus d'Abousir). Traduction et commentaine, BdE 65 (Kairo 1976), S. 565-588 und A. M. Roth, Egyptian Phylae in the Old Kingdom. The Evolution of a System of Social Organisation, SAOC 48 (Chicago 1991).

31 Letzte umfassende Bearbeitung durch G. P. van den Boorn, The Duties of the Vizier. Civil Administration in the Early New Kingdom (London/New York 1988).

${ }^{32}$ Zur generellen Datierung der Dienstaufgaben des Wesirs ist vor allem J.M. Kruchten in seiner Rezension zu van den Boorns Publikation, BiOr 48 (1991), 826-831 zu vergleichen, der die Ansetzung des Archetyps im späten Mittleren Reich aus gutem Grund favorisiert.

${ }^{33}$ Siebzig Tage waren die übliche Länge einer ägyptischen Mumifizierung. 
die Gouverneure, die den Gott bei den Prozessionen eintreten lassen und zur Erscheinung bringen. Das Neujahrsfest sowie die jahreszeitlichen Feste werden explizit genannt.

Neben diesen religiösen Pflichten steht der administrative Aufgabenbereich der Gouverneure. Als Vorsteher der Priester haben sie die Tempelschreiber unter ihrer Autorität, speziell in Schatzhaus, Scheune, Viehstall und Geflügelhof des jeweiligen Stadtgottes sind ihnen unterstellte Schreiber tätig, die mit den Schreibern des Königs und des Wesirs zusammenarbeiten sollen. Entsprechend erhalten die Gouverneure auch Berichte über erfolgreiche und mißlungene Dienstmissionen, wobei der Fähige von ihnen belohnt, der Unfähige getadelt werden soll. Das Tadeln beinhaltet auch ganz konkrete Strafen, als Möglichkeiten genannt werden Schläge, Haft und Gütereinzug. Wenn derartige Maßnahmen nötig sind, soll der Delinquent in die Residenz gebracht werden, bzw. die Gouverneure erhalten aus der Residenz Dekrete und Nachrichten und erstatten dem Palast Meldung. Offenbar sollen sie dreimal jährlich zu festlichen Anlässen in die Residenz kornmen. Sobald die Amtsträger alt geworden sind, sollen sie, sofern der etwas fragmentierte Text richtig verstanden ist, "pensioniert" werden, $d$. h. einen Aufenthalt im Vestibül des Palastes erhalten, wo sie mit allem Nötigen versorgt werden und keiner schlechten Behandlung durch hohe Beamte ausgesetzt sein sollen. Im Todesfall werden sie nicht nur einfach in die Balsamierungswerkstatt gegeben, sondern es wird speziell angegeben, daß sie Salbungen erhalten sollen, auch Textilien, welche die ${ }_{n}$ beiden Gefährtinnen ${ }^{\prime}$ (d. h. Isis und Nephthys) gewebt haben, sowie Textilien aus abgelegten Stoffen der [Götterstatuen] werden ihnen bestimmt. Nicht nur soll ihr Sohn in ihr Amt eingesetzt werden, sondern sie erhalten auch eine Statue (twt.w) im Tempel.

Als Anhang zur Sektion über die Gouverneure findet sich ein Exkurs über das Pantherfell, das die Gouverneure (und auch andere Priesterränge) bei bestimmten Anlässen tragen. Es soll keineswegs ein echtes Pantherfell sein, vielmehr wird es aus Stoff hergestellt und mit Edelsteinen und Gold besetzt. Keinesfalls soll Leder ihren Leib berühren. In der Praxis gibt es tatsächlich Belege dafür, daß Pantherfelle aus Stoff imitiert wurden, so fanden sich konkrete Exemplare im Grab des Tutancharnun (allerdings neben echten Pantherfellen), ${ }^{34}$ im Grab TT $32^{35}$ sowie ein Stück unsicherer Herkwnft im Metropolitan Museum of Arts New York. ${ }^{36}$ Das Ledertabu dürte mit dem Wolltabu für ägyptische Priester zusammenhängen, das auch sonst, vorwiegend aus griechischen Quellen bekannt ist. ${ }^{37}$

Exheblich schlechter erhalten ist leider die Sektion über die Propheten des Tempels. Thre Tätigkeiten konzentrieren sich auf die Rituale, die Details bleiben aber weitgehend im Dunkeln. Auch sie erhalten eine Statue (bnti). Mehrere weitere Sek-

34 Briefliche Auskunft von Dr. G. Vogelsang-Eastwood, der ich dafür herzlich danken möchte.

${ }^{35}$ L. Kákosy, Dzsehutimesz súrja thébánan Budapest 1989), S. 51 Abb. 54.

36 Den Hinweis hierauf verdanke ich ebenfalls Vogelsang-Eastwood. Das Stück ist erwähnt bei H. E. Winlock, The Costume of an Egyptian Priest, BMMA 27/8 (August 1932), S. 186 sowie Th. Logan, Papyrus Harkness, in: Fs Hughes, SAOC 39 (Chicago 1976), S. 148.

3? Wichtigste Quelle hierfür ist Plutarch, De Iside Kap. 4, wo speziell die Begründung geliefert wird, daß Wolle, Haar und Nägel aus überschüssiger Materie entstënden (352 CD); weitere Angaben finden sich etwa Herodot, 2, 81 sowie im Gnomen des Idios Logos, s. J. G. Griffiths, Plutarchs De Iside (Swansea 1970), 270 f. (Kommentar); A. Lloyd, Herodotus, Book II. Commentary 1-98, EPRO 43 (Leiden 1976), S. $342 \mathrm{f}$. 
tionen mit Priestern spezieller Ritualtätigkeit sind so schlecht erhalten, daß die reine Identität des Amtes einstweilen unsicher ist. Dabei dürften unter anderem diejenigen genannt sein, die sich speziell um osirianische Rituale kümmern, jedenfalls werden einige Phrasen aufgegriffen, die sich ähnlich bereits in der oben erwähnten Sektion über Zugangsberechtigung zu osirianischen Bereichen der Tempelanlage finden.

Ein klareres Bild gewinnt man erst wieder bei der Behandlung des Schreibers des Gottesbuches (sk3.w m\̧3.t nćr). ${ }^{38}$ Er arbeitet recht eng mit dem Priester der Sachmet und dem Skorpionsbeschwörer zusammen, so daß es viele Aktivitäten gibt, die in diesen drei aufeinander folgenden Dienstanweisungen mehrfach genannt werden. Zunächst erfahren wir von Ächtungsritualen, welche der Schreiber des Cottesbuches gegen Apopis und die Kinder der Ermattung ${ }^{39}$ durchführen soll. Verwendet werden die üblichen Schriftträger bzw. Figurinenmaterialien Ton, Akazien- und Mandelholz, vermutlich auch Papyrusrollen und Wachs.

Sehr interessant ist die Beschreibung, daß der Schreiber des Cottesbuches das Personal des Tempels auf Aussatz ${ }^{40}$ inspizieren soll. Im Verein mit den weiteren Angaben, die beim Skorpionsbeschwörer noch gemacht werden, läßt sich erschlieBen, wie die Ägypter streng auf mögliche Fälle von Aussatz achteten, die infizierten Personen aus der Gemeinschaft ausschlossen und Orte, wo Fälle aufgetreten sind, gereinigt haben. Vor diesem Hintergrund gewinnen die von klassischen Autoren im Zusammenhang mit dem Exodus der Juden angeführten Traditionen über eine vertreibung von Aussätzigen ${ }^{41}$ eine etwas andere Dimension.

Ebenfalls kontrolliert werden sollen die Tiere, die in die Viehhürde bzw. zur Schlachtbank kommen, wobei der Schreiber des Gottesbuches mit dem Personal der Bibliothek ${ }^{42}$ (č.t pr-inh) sowie dem Sachmetpriester zusammenarbeitet. Dabei ist ein doppelte Wirkung beabsichtigt. Zum einen sollen Tiere geschützt werden, die ihren körperlichen Markierungen nach als heilige Tiere anzusehen sind, zum anderen - hierfür ist schwerpunktmäßig der Sachmetpriester zuständig - sollen infizierte und nicht zum Verzehr geeignete Schlachttiere erkannt werden.

Gewissermaßen als Anhang hinter der Nachfolgeregelung, die sonst jeden Abschnitt abschließt, wird noch angegeben, der Schreiber des Gottesbuches solle Schriften abfassen über das, was er im Tempel tut, außerdem u. a. bei der Herstellung von Götterstatuen im Goldhaus anleitend teilnehmen.

Der Sachmetpriester, der unbeschadet seines Namens nicht an Kulte der Sachmet

${ }^{38}$ Für diesen und die beiden folgenden Abschnitte verweise ich auf eine detailliertere Beschreibung in J. F. Quack, Between Magic and Epidemic Control. On Some Instructions in the Book of the Temple, in: S. Seidlmayer (Ed.), Religion in Context. Imaginary Concepts and Social Reality (i. Dr).

39 "Kinder der Ermattung ${ }^{\circ}$. (msj.w błs $)$ ist ein gängiger ägyptischer Begriff, der mutmaßlich eine bewußte Verzerrung von „Kinder der Rebellion“" (msj.w bly ) darstellt.

${ }^{40}$ Das im Ägyptischen verwendete Wort ist $h m w . t-s 5$, zur Bedeutung s. die Bemerkungen von J. Osing, Hieratische Papyri aus Tebtunis I, S. 115 Anm. e) sowie J. F. Quack, BiOr 54 (1997), Sp. 332 .

${ }^{41} \mathrm{Zu}$ diesen Traditionen s. zuletzt J. Assmann, Ägypten. Eine Sinngeschichte (München/Wien 1996), S. 255-258; 440-446; Th. Schneider, Ausländer in Ägypten während des Mittleren Reiches und der Hyksoszeit, Teil 1. Die ausländischen Könige. ÄAT 42 (Wiesbaden 1998), S. 76-98.

${ }^{42}$ Die etwas behelfsmäßige Übersetzung „Bibliothek“ verwende ich bewußt, um der üblichen, aber nach der Vokalisation sehr problematischen Auffassung als „Lebenshaus“ zu entgehen. 
gebunden, sondern überregional üblich ist, ${ }^{43}$ soll eine Reihe von Schutz- und Reinigungsritualen durchführen, unter anderem natïrlich die Besänftigung der Sachmet, aber auch den ${ }_{n}$ Schutz des Hauses ${ }^{4}$. Eine besondere Betonung erhalten Besänftigungsrituale, die dreimal jährlich, nämlich am Neujahrstag (wpi-rnp.t), im ersten und im vierten Monat der Sommerjahreszeit ( $t p l s m w 3 b t 4$ smw) durchgeführt werden sollen. Bei ihnen soll ein großes Opfer in Sepat, in Memphis und im Binsengefilde dargebracht werden. Ferner ist der Sachmetpriester für Hygiene, ${ }^{44}$ nämlich für das Reinigen der Cefäße im Tempel zustöndig. Besonders beachtenswert ist sein Wirken im Seuchenschutz, indem er nämlich Himmel und Erde auf Anzeichen von Pestilenz an Mensehen und Vieh untersucht. Im Zweifelsfall soll ein Komitee von zwei Sachmetpriestem, dem ältesten Königssohn und dem Wesir darüber Meldung in der Residenz erstatten. Wie schon oben erwähnt, arbeitet er mit dem Schreiber des Gottesbuches zusammen, um Vieh zu mustern.

Dèr Skorpionsbeschwörer erweist sich als der typische ägyptische Magier, zumindest was denjenigen Bereich der Magie betrifft, der sich mit Schutz vor Tieren und der Heilung von Krankheiten beschäftigt, außerdem wenigstens teilweise auch mit Schadensmagie. Für letzteren Bereich soll er das Buch zum Niederwerfen des Apopis sowie der Feinde des Osiris und des Königs rezitieren.

Darüber hinaus soll er die Anzeichen für die Nilüberschwemmung zusammen mit den Mitarbeitem der Bibliothek des Königshauses, die im Tempel sind, sowie dem ältesten Königasohn beobachten. Wohl im Zusammenhang damit steht, daß er in Sepa, in der Residenz, in Heliopolis, in den Sumpfmarschen, in Babylon und im Binsengefilde das Nilbuch ins Wasser werfen soll. Ebenso sollen das Strömen der Überschwemmung und die damit verbundenen Verlagerungen des Flußlaufes bzw. Gebietsveränderungen gemeldet werden.

Zurück zur Magie führt die Angabe, er solle die Schlangen mustern und die schädlichen im Gau vertreiben, entsprechend auch Schutzamulette gegen Schlangenbiß, Löwen und andere gefährliche Tiere herstellen sowie widrige Winde bei der Barkenfahrt des Gottes verhindern. Die Ausdrucksweise des. Textes legt es nahe, daß ein Bezug zu den sehr häufigen sogenannten Texten A und B der Horusstelen besteht.

Wie schon der Schreiber des Gottesbuches, soll auch er auf Aussatz kontrollieren, im Gegensatz zu ihm aber die gesamte Bevölkerung, wobei auch die Schutzmaßnahmen stärker betont werden. Im Zusammenhang damit steht, daß er generell als Arzt bei bestimmten Krankheiten wirken soll. Soweit diese lexikalisch einigermaBen faßbar sind, handelt es sich stets um Hautkrankheiten bzw. Krankheiten, die sich auf der Haut merklich äußern.

Es folgt eine wenig bekannte Gestalt mit dem Titel nsw.ti. Der betreffende Mann ist speziell für den Königskult im Tempel zuständig. Konkret soll er den Königsstatuen, die sich im Tempel und in den Kapellen sowie im Umgang befinden, Opfergaben darbringen und dem Königska huldigen.

Recht ausführlich behandelt wird der Schulmeister ( $l m l-r \xi s b 3 . w)$. Er soll die Kinder der Propheten, der Vorlesepriester und der hochrangigen Priester unterrich-

43 Hierzu und zum folgenden Abschnitt vgl. die ausführliche Bearbeitung durch F. von Känel, Les prêtres-ouâb de Sekhrnet et les conjurateurs de Serket (Paris 1984).

*t Vgl. hierzu und rum folgenden H. Engelmann, J. Hallof, Der Sachmetpriester. Ein früher Repräsentant der Hygiene und des Seuchenschutzes, SAK 23 (1996), S. 103-146. 
ten und unter ihnen denjenigen auswählen, der seinen Fähigkeiten nach seinem Vater nachfolgen soll. Wert gelegt wird, im Zusammenhang des Tempelkultes verständlich, auf eine musikalische Ausbildung in bestimmten Gesangstechniken. Auch alle sonstigen Verhaltensweisen im Tempel sollen unterrichtet werden. Úber diesen Bereich, sozusagen die "Grundausbildung" hinaus, ertält der Schulmeister noch einen speziellen Auftrag, die Kinder der Propheten ${ }^{45}$ unterrichten. Dabei werden vier verschiedene Ausbildungsphasen unterschieden. An erster Stelle steht die Unterweisung in den „Gottesworten" (mtw-nčr), den Gebräuchen von Ober- und Unterägypten und den Spezifika des betreffenden Gaues, d. h. den in den gauspezifischen Monographien niedergelegten res sacrae und Lokalmythen, sowie den Vorschriften für den Königshof.

In der zweiten Phase geht es u. a. um die Eimprägung der Festrituale und der Kultausübung sowie die Erklärung von schwierigen Textstellen. Der dritte Unterrichtsbereich umfaßt medizinische Kenntmisse, der vierte die Ekliptikomina, Schriften des Balsamierungshauses $(p r-n f r)$ und einige schlecht erhaltene Bereiche.

Der als nächster behandelte Titel ist zwar in einer kleinen Lücke verloren, dem Inhalt nach handelt es sich jedoch mit einiger Wahrscheinlichkeit um den Umrißzeichner (sh3.w kt), der eine Reihe von Handwerksberufen einleitet. Er soll die Mauern der Gottesschreine nach den Modellvorlagen des Bücherhauses bemalen. Außerdem sollen Bereiche des Tempels ${ }^{46}$ am 16. Tag des ersten Monats der Überschwemmungszeit mit Ocker gefärbt werden. Bei der Herstellung der Statuen, die unter Anleitung des Vorlesepriesters und des Personals der Bibliothek stattindet, kommt er vor den Bildhauern zum Einsatz. Darüber hinaus soll er alles, was in den Tempeln hergestellt wird, mit dem Namen seiner Majestät und der Gaugötter markieren, speziell Textilien, die für den Gott bestimmt sind. Selbstverständlich kommt er auch beim Ritual der Mundöffnung zum Einsatz ${ }^{47}$ und zwar ebenso für Statuenherstellung im Goldhaus wie für die funeräre Nutzung des Textes.

Es folgt der Bildhauer ( $k s . t i g n w . t i)$. Über seine prinzipielle Arbeit bei der Statuenherstellung hinaus läßt der zunehmend schlechtere Erhaltungszustand leider wenig Schlüsse zu. Gleiches gilt für den Oberhandwerker (wr hmw,w) und den „Obersten der Oberen" (hri-hri.w). ${ }^{48}$

Für einen längeren Bereich ist dann keine gesicherte Abfolge zu erstellen, da eventuell dorthin gehörige Fragmente keine Seitenzahlen aufweisen. Ein denkbarer Kandidat für diesen Bereich ist etwa ein hohes, aber relativ schmales Fragment, in dem vor allem die Prospektoren des Tempels (smn.tiw) erwähnt werden. Sie sollen auf der Suche-nach wertvollen Mineralien in die Wüste ziehen und bis zu den Oasen der Westwïste gelangen.

${ }^{45}$ Die sonstigen Ränge sind hier nicht aufgefïhrt, was darauf hindeutet, daß es sich um eine exklusive Zusatzqualifikation für die Kinder der höchsten Würdenträger handelt.

46 Die genaue Angabe ist leider einer Lücke zum Opfer gefallen.

${ }^{47}$ Dazu ist zu bemerken, daß ein bislang unklarer Titel unter den Handwerkern des Mundöffnungsrituals tatsächlich entweder direkt $s t 3{ }^{3} \mathrm{kf}$ zu lesen ist oder zumindest sein sachliches Äquivalent darstellt, da er an einer Stelle in der demotischen Version des Buches vom Tempel mit $s h 3 k t$ in zweifelsfreier Schreibung wiedergegeben wird.

${ }^{48} \mathrm{Zu}$ diesern Titel, der vorwiegend bei Steinbruchexpeditionen des Mittleren Reiches belegt igt, 8. ausführlich K.-J. Seyfried, Beiträge zu den Expeditionen des Mittleren Reiches in die Ost-Wüste, HÄB 15 (Hildesheim 1981), S. 33-35. 
Ein durch Seitenzahlen fixierter Bereich ab Seite 20 der Haupthandschrift nennt hauptsächlich Ämter praktischer Tätigkeit. Zunächst erscheint der Küchenmeister (Irl-psi). Er ist erwartungsgemäß für die Lebensmittel zuständig, die angesichts der zahlreichen Opfer im Tempel kein kleiner Bereich sind.

Es folgt der für den Brandopferaltar Zuständige ( $\left.l i l-b 3 w^{\prime} l\right)$. Er ist mit den Brandopfern $(b a w t n t h .5 w l)$ beschäftigt. Daneben soll er auch generell in seinen Zugangsbereichen Feuer entfachen und auslöschen.

Der nachfolgende Amtsträger, dessen Titel leider in einer kleinen Lücke verloren ist, scheint vorrangig dafür verantwortlich, die Ordnung aufrecht zu erhalten. So soll er bei Festen einen freien Weg bahnen ( $\breve{s} s$ w3ik.t) oder die Türhüter anweisen, damit niemand ohne Erlaubnis zur Tür hereinkommt. Anschließend, ohne daß der konkrete Übergang erhalten ist, scheint es um verwaltungsmäßige Abrechnungen zu gehen, bei denen der Besitzstand des Tempels in Schatzhaus, Scheune und Viehhürde zu kontrollieren ist. Der betreffende Amtzinhaber soll mit dem Lesonis-Priester und den beiden Gouverneuren zusammenarbeiten.

Wieder erhalten ist der nächste Titel, nämlich der Opfertischschreiber (sb3.w wth.w). Dafür ist seine Dienstanweisung nur sehr bruchstückhaft erhalten. Vom darauffolgenden, wieder in einer Lücke verlorenen Amtsträger erfährt man immerhin, er sei dafür zuständig, die Kultgeräte des Tempels (hnw $m$ țbh [...]) auszuhändigen, wobei jeweils beim Phylenwechsel die vier Vertreter der einen Phyle sie den Vieren der nächsten übergeben würden. Ohne daß klar wird, wer hiẹr zuständig ist, wird dann u. a. noch die Übergabe von Textilien, Halskragen und Kränzen erwähnt.

Als nächstes faßbar werden die Hirten des Tempels. Man erfährt unter anderem, wie die Milch durch ein großes Sieb (bnm.t wr.t) geseiht werden soll. Nach einigen sehr schlecht erhaltenen Bereichen wird der Schlachter (hn.ti) genannt. Unter anderem soll er ein im Text des Buches vom Tempel mehrfach erwähntes Tier namens (i) $s h 3$ schlachten, dessen Identifizierung noch Schwierigkeiten bereitet.

Als letzter Titel in diesem Textbereich ist noch der Rindervorsteher (imi-r? ih.w) zu erwähnen. Bei ihm handelt es sich wieder um eines derjenigen Ämter, die nicht der Rotation unterliegen, sondern mit zwei mutmaßlich permanent tätigen Personen besetzt werden. Von ihnen ist der eine als Horus, der das Herz prüft ( $h r . w$ l $p$ ib) bezeichnet, der andere ist micht erhalten, mutmaßlich aber als Anubis, der das Herz prüft, zu ergänzen. ${ }^{49}$

Der oben bereits kurz erwähnte Lesonispriester ( $i m l-r 3$ šnw) erscheint übrigens tatsächlich mit Resten seiner Dienstanweisung in einem Fragment, das etwa in diesen Bereich gehören dürfte. Er folgt dabei in der Abfolge der Titel auf den Imker (lri-bi.t). Bemerkenswerterweise soll auch der Lesonis der üblichen Rotation mit vier sich monatlich abwechselnden Vertretern unterliegen. Diese Angabe sowie seine vergleichsweise wenig prominente Rolle stehen eher im Gegensatz zu dem, was wir aus der Spätzeit über den Lesonis und seine wichtige Sonderposition gegenüber den sonstigen Priestern wissen.

Die letzten Bereiche des Buches vom Tempel werden zunehmend fragmentari-

49 $\mathrm{Zu}$ diesen Göttergestalten s. vor allem A. Egberts, In Quest of Meaning. A Study of the Ancient Egyptian Rites of Consecrating the Meret-Chests and Driving the Calves, EU 8, (Leiden 1995), S. 306; einige Bemerkungen auch bei J. F. Quack, A New Bilingual Fragment from the British Museum (erscheint in IEA). 
scher und schwerer zu plazieren. Erwähnenswert ist hier etwa ein Fragment, in dem Personen genannt sind, die vi. a. die Nekropole kontrollieren sollen. Die Begräbnisstätten der heiligen Tiere und der Propheten spielen dabei eine besondere Rolle. Außerdem sollen sie darauf achten, daß man in bestimmten heiligen Bereichen keinen Urin oder Kot hinterläßt. Im Falle eines Verstoßes werden harte Strafen angedroht, offenbar u. a. Abschneiden von Nase und Ohren.

Unter den niederen Bediensteten des Tempels, die nur in kleinen und fragmentarischen Bruchstücken faßbar sind, möchte ich noch den Viehmäster oder den Fallensteller erwähnen. Besonders zahlreich vertreten sind Leute, die mit Getreide zu tun haben, d. h. es unter anderem mahlen müssen. Hier werden nicht weniger als fünfzig Mann pro Monat, insgesamt also zweihundert angesetzt.

Ein etwas umfangreicher erhaltener Bereich des Textes spricht unter anderem

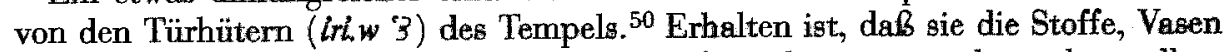
und sonstigen Geräte des Tempels in ihren Aufbewahrungsorten bewachen sollen. Hirten sollen den als ${ }_{n}$ Gott ${ }^{*}$ bezeichneten. Bewohner des Bezirks der heiligen Tiere $(m 3 r w)$ bewachen, speziell wenn er ins Feld auszieht. Es scheint vor allem an heilige Stiere gedacht zu sein. Ferner erfährt man auf dem betreffenden Bruchstück nochmals von Schutzmagie zum Verschließen des Mundes von Schlangen und zur Abwehr von Schlangen. ${ }^{51}$ Auch Ächtungsrituale mit Hilfe von Feindfiguren werden erneut erwähnt.

Bisher war immer von männlichem Tempelpersonal die Rede, es gibt jedoch auch einige Hinweise auf weibliche Teilnahme am Kult. Zum einen werden die Sängerinnen erwähnt. ${ }^{52}$ Darüber hinaus gibt es einige weitere, leider meist sehr unklare Bemerkungen in schlecht erhaltenen Bruchstücken. Potentiell besonders bemerkenswert ist dabei eine Angabe über Reinheitsvorschriften für eine Frau, der eine Frist gesetzt wird. Zudem wird im Falle eines Makels ( $b w$ ) leider nicht genauer definierter Art mit der Amtsenthebung (tr $m$ wnw, $t$ ) gedroht.

Schließlich sollte als einstweilen problematischer Bereich noch die Beschreibung eines großen Festes mit zahlreichen Opfern erwähnt werden. Der Sache nach besteht einige Wahrscheinlichkeit, daß es sich um das Neujahrsfest handelt. Seine Position im Text bleibt derzeit völlig offen. Auch seine Zugehörigkeit als solche möchte ich nur akzeptieren, da es von diesem Bereich Bruchstücke verschiedener Handschriften gibt, von denen zumindest die eine in einem Duktus geschrieben ist, der nur für die betreffende Handschrift des Buches vom Tempel selbst belegt ist.

Abschließend zum Buch vom Tempel sei hier noch auf die Möglichkeit hingewiesen, daß ein kleiner Teil davon schon lange in einem hieroglyphischen Auszug bekannt ist. Dabei habe ich eine Inschrift im Goldhaus des Tempels von Dendera im Auge (Dendera VIII 128, 15f.; 131, 1-6), die in neuerer Zeit öfters kommentiert

${ }^{30}$ Diese Türhüter entsprechen, wie von F. Hoffmann und J. F. Quack in einem in Yorbereitung befindlichen Artikel im Detail nachgewiesen wird, den Pastophoroi der griechischen Texte.

${ }^{51}$ Hier sei daran erinnert, daß die wichtige magische Statue des Djedhor, des Retters, Edition von E. Jelínková-Reymond, Les inscriptions de la statue guérisseuse de Djed-Her-Le-Sauveur, BdE 23 (Kairo 1956), einem Türhüter gehört.

52 Sie finden sich auf dem bereits veröfentlichten pTebtunis Tait 32, Edition W. J. Tait, Papyri from Tebtunis in Egyptian and Greek, TE 3 (London 1977), S. 88, T. 7; das betreffende Stück gehört mit pCarlsberg 316, pBerlin 14439c) sowie einem unveröffentlichten Papyrus in Florenz zu einer Handschrift. 
worden ist. ${ }^{53}$ Sie enthält Dienstanweisungen für die Handwerker, die in ihrer Struktur und ihrem Stil den Dienstanweisungen des Buches vom Tempel sehr ähneln. Die Verwandtschaft zeigt sich auch darin, daß im Gegensatz zum realen $\mathrm{Zu}$ stand zur Zeit der Niederschrift mit nur vier Phylen operiert wird. Bisher konnte leider noch keine konkrete Texrübereinstimmung mit einer Handschrift des Buches vom Tempel nachgewiesen werden. Aber immertin ist in Florenz inzwischen ein kleines Fragment aufgetaucht, von dem zumindest erkennbar ist, daß es das Personal des Coldhauses nennt.

Neben dem eigentlichen Buch vom Tempel sind bei der Textaufnahme eine Reihe weiterer Texte aufgefunden worden, die engere Berührungspunkte zu manchen inhaltlichen Bereichen zeigen und hier wenigstens kurz vorgestellt sein sallen.

Ein aus Deir el-Bahri stammendes römerzeitliches Papyrusfragment im British Museum (pBM 10565) ${ }^{54}$ weist mehrere Ähnlichkeiten zur historischen Einleitung des Buches vom Tempel auf. Wie dort wird der Tempel des Atum von Heliopolis genannt, ebenso findet sich die Erwähnung von sieben Jahren im Zusammenhang mit der Nilüberschwemmung. Allerdings scheinen hier nicht nur sieben magere, sondern auch sieben fette Jahre genannt. Schließlich erscheint statt des Hordjedef ein anderer berühmter Weiser, nämlich der oberste Vorlesepriester Imhotep. Der sehr fragmentarische Erhaltungszustand des kleinen Stückes macht genauere Schlußfolgerungen fast unmöglich, die Sprache des Textes ist, soweit nachprüfbar, Mittelägyptisch.

Mit einiger Wahrscheinlichkeit vom Buch des Tempels abzutrennen ist eine Sammlung von Dekreten, die angeblich von Königen des Alten Reiches verfaßt wurden. Im Text konkret erhalten ist einmal Cheops, und zwar in der ausführlichen Form seines Names als $h n m$ w $w w i=f w(l)$. Daneben wird auch Djoser erwähnt, und neben ihm erscheint der oberste Vorlesepriester Imhotep, Sohn des Ptah. In den Dekreten wird unter anderem der Aufbau von Tempeln im jeweiligen Gebiet des Empfängers angeordnet. Dabei sollen auch Handwerker aus der Residenz ausgeschickt werden, um die große Namenstitulatur des Herrschers einzugravieren. Bestimmte Dekrete scheinen an alle Hauptzentren in Ägypten gerichtet zu sein und die jeweiligen spezifischen Priester und Priesterinnen aufzufordern, in die Residenz zu kommen. Historisch bemerkenswert dürfte eine Erwähnung des Fürsten von Byblos sein.

Vom selben Schreiber, dem wir auch die besterhaltene Handschrift des Buches vom Tempel (pCarlsberg 313) verdanken, stammt auch ein zweiter Papyrus, der sich aber durch deutlich geringere Blatthöhe und Zeilenzahl bei großen Fragmenten leicht abtrennen läßt. Die Zuweisung kleiner Bruchstücke kann allerdings Schwierigkeiten bereiten, da es um ein verwandtes Thema geht, nämlich Regelungen für den Tempelbetrieb. Diese Handschrift enthält zum einen Gesetze, und zwar

53 Bearbeitungen und Bemerkungen bei E. Chassinat, Le mystère d'Osiris au mois de Khoiak (Kairo 1966-68), S. 481f.; F. Daumas, Quelques textes de l'atelier des orfevres dans le temple de Dendara, in: Livre du Centenaire 1880-1980, MFAO 104 (Kairo 1980), S. 109-118 (dort S. 112-114); S. Cauville, Les statues cultuelles de Dendera d'après les inscriptions pariétales, BIFAO 87 (1987), S. 73-117 (dort S. 110f); Ph. Denchain, L'atelier des Orfevres à Dendara et les origines de l'alchimie, CdE 65 (1990), S. 233 f.; S. Aufrère, L'univers minéral dans la pensée égyptienne, BdE 105 (Kairo 1991), S. 363; D. Kurth, BiOr 51 (1994), Sp. 325 f.

54 Das Stück stammt aus den Grabungen von E. Naville zu Anfang des Jahrhunderts. 
anscheinend Strafgesetze, für Vergehen im Tempelbereich, z. B. „Jederman aher, der ertappt wird, wie er Vögel gestellt oder [Fisehe] gefangen hat [...]. ${ }^{455}$ Die verhängten Strafen fallen durchaus hart aus. Erwähnt werden etwa die Amtsenthebung im Tempel ( tr $m$ wnw.t), Schläge mit einem als ${ }_{n} H_{u n d}$ " bezeichneten Objekt (vermutlich eine Art Peitsche) sowie die Pfählung ( auch Speisetabus bestimmter Priester sowie Bekleidungsvorschriften und generelle Anweisungen für das Verhalten (tp-rt). Ein Beispiel ist etwa ${ }_{n}$ Nicht soll irgendein Textil hineinkommen zum (Bereich der) großen Reinheit, ausgenommen Lendenschurz(?) ( $s \zeta^{\zeta} w$ ) und Gürtel(?) ('gs)" oder ,Wenn er zum Tempel kommt, wenn er den Pronaos des Tempels erreicht, soll er die weißen Sandalen ${ }^{56}$ ablegen ${ }^{4}$ und ähnlich „Nicht treten weiße Sandalen ein in irgendeinen Gottesschrein in Himmel, Erde und Unterwelt des Tempels." Leider in weitgehend verlorenen Zusammenhang erscheint der Name des Königs Djoser.

Ein sehr eigener Text schließlich, dessen ausführliche Würdigung an anderer Stelle erfolgen muß, ist vorwiegend als Dialog zwischen Pharao und Imhotep stilisiert. Dabei geht es vor allem um den Tempel und seine Architektur in ihrer symbolischen Ausdeutung. Astronomische bzw. astralmythologische Gesichtspunkte scheinen eine entscheidende Rolle zu spielen. ${ }^{57}$ Bemerkenswert ist übrigens, daß ein dem Duktus nach sicher zugehöriges Fragment Reste einer Kosmogonie "hermopolitanischen “ Typs enthält und damit eine bemerkenswerte Ähnlichkeit zu den sogenannten „Fragmenten memphitischer Theologie in Demotischer Schrift (pBerlin 13603) aufweist, in denen Imhotep auch eine nicht geringe Rolle spielt. ${ }^{58}$

\section{Summary}

The "Book of the Temple" is a large manual on the ideal Egyptian temple that the author is currently reconstructing from forty mostly unpublished papyri. The book was originally composed in middle Egyptian, but several manuscripts attest a translation into Demotic, the vernacular language of the late period. The present article is a preliminary report. After a short introduction providing a fictive historical setting for the text in the early dynastic period, the text describes the architectural layout of the ideal Egyptian temple in great detail. The book then turns to general rules for priests. They include an oath formula with a list of sinful deeds,

${ }^{55}$ Zumindest in bestimmten Bereichen der Tempelanlage, vor allem in einer Zone um das Osirisgrab, war der Fang von Fischen und Vögeln verboten, s. etwa H. Junker, Das Götterdekret über das Abaton, DAWW 54 (Wien 1913), S. VIf., 31 u. 72.

${ }^{56} \mathrm{Zu}$ weißen Sandalen in Ägypten s. zuletzt St. Schwarz, Zur Symbolik weißer und silberner Sandalen, ZÄS 123 (1996), S. 69-84.

${ }^{57}$ Hier sollte man an die auch sonst bezeugte Tradition der Spätzeit denken, Imhotep (bzw. in griechischsprachigen Texten Asklepios) als großen Astrologen anzusehen. Tatsächlich werden ihm in unpublizierten Handschriften auch einige demotische astrologische Traktate zugeschrieben.

58 Edition einiger Fragmente bei W. Erichsen, S. Schott, Fragmente memphitischer Theologie in demotischer Schrift (Pap. demot. Berlin 13603), AAWLM Mainz (Wiesbaden 1954). Eine Reihe weiterer Bruchstücke des (aus Kartonage gewonnenen) Papyrus liegen noch unveröffentlicht in Berlin. 
which the priest will not commit or has not committed. A final section assigns detailed duties to each priest and temple employee. The article mentions briefly several related compositions. The mentioned oath is already known from two Greek papyri, on the basis of which earlier studies have pointed to the use of textually similar nogative confessions in the mysteries of Isis and to their reception in Roman literature. 